Int. J. Odontostomat., 6(1):105-110, 2012.

\title{
Maltrato Infantil: Actitud y Conocimiento de Odontólogos en Concepción, Chile
}

\author{
Attitude Towards Child Abuse According to the \\ Knowledge of Dentists in Concepción, Chile
}

Claudia Fierro Monti"; Engel Salazar Salazar ${ }^{* *}$ : Lucía Ruiz Salazar ${ }^{* *}$ : Luis Luengo Machuca ${ }^{* * *}$ \& Antonieta Pérez Flores ${ }^{*+* *}$

FIERRO, M. C.; SALAZAR, S. E.; RUIZ, S. L.; LUENGO, M. L. \& PÉREZ, F. A. Maltrato infantil: actitud y conocimiento de odontólogos en Concepción, Chile. Int. J. Odontostomat., 6(1):105-110, 2012.

RESUMEN: El maltrato infantil (MI) es considerado un problema de salud pública mundial, ocurre en todos los países y en todos los grupos sociales y religiosos. El trabajo de los odontólogos implica la atención de gran cantidad de niños y en sesiones sucesivas lo que pone al profesional en un lugar privilegiado para la detección del MI. El propósito de esta investigación fue describir el conocimiento y la actitud frente al MI de los odontólogos pertenecientes a los nueve centros asistenciales de salud de Concepción, Chile. Se realizó un estudio exploratorio de enfoque cuantitativo, descriptivo y transversal. Se confeccionó una encuesta que recogió datos demográficos, conocimiento, actitud, sospecha y denuncia sobre MI. La población de estudio consistió en 53 odontólogos. Las pruebas estadísticas utilizadas fueron Chi cuadrado y Wilcoxon (Mann-Whitney U). En general el conocimiento es satisfactorio. Del 43,3\% que declara haber sospechado alguna vez sobre casos de MI, sólo el $56,5 \%$ lo ha denunciado. Se encontró diferencia significativa (p: 0,0326 ) al relacionar las variables de género y conocimiento, siendo mayor en las mujeres. Sin embargo entre las variables género y actitud no hubo diferencia. Las variables: origen de la información recibida, número de años de trabajo, número de niños atendidos a la semana y estudios de postgrado resultaron independientes con respecto al númerode respuestas correctas de conocimiento. El origen de la fuente de información no se observó en la formación de pregrado de los profesionles, por esto se plantea entonces la necesidad de incorporar o reforzar el tema del Ml en las asignaturas de pregrado en las Facultades de Odontología de la región.

PALABRAS CLAVE: maltrato infantil, conocimiento, actitud, odontólogos.

\section{INTRODUCCIÓN}

Existen distintas definiciones de Maltrato Infantil (MI). La Ley Chilena No 19.324 de violencia familiar lo define como el resultante de una acción u omisión que produzca menoscabo en la salud física o psíquica de los menores. La UNICEF lo entiende como los niños/as y adolescentes de hasta 18 años que "sufren ocasional o habitualmente actos de violencia física, sexual o emocional, sea en el grupo familiar o en las instituciones sociales". Sin embargo, la definición más completa y específica la da la OMS definiéndolo como los abusos y la desatención de que son objeto los menores de 18 años de edad, e incluye todos los tipos de maltrato físico o psicológico, abuso sexual, desatención, negligencia y explotación comercial o de otro tipo que causen o puedan causar un daño a la salud, desarrollo o dignidad del niño.

La prevalencia mundial de MI varía según la definición con la que se mida. Las definiciones más amplias dan tasas de prevalencia más grandes (Jouriles et al., 2008). UNICEF (2008) en su tercer estudio sobre MI, en el cual se realizó una encuesta a 1525 niños/as que cursaban octavo de enseñanza básica concluyó que el $75 \%$ de los niños y niñas su-

Profesor Asociado Facultad de Odontología, Universidad de Concepción, Chile, Especialista en Odontopediatría Universidad de Concepción, Chile. Doctoranda Universidad de Córdoba, Argentina.

* Estudiantes Quinto Año Facultad de Odontología, Universidad de Concepción, Chile.

*** Magister en Estadística Aplicada, Universidad de Concepción, Chile.

**** Profesor Asistente Facultad de Odontología, Universidad de Concepción, Chile, Magister Universidad Complutense, España, Doctoranda Universidad de Córdoba , Argentina. 
fre violencia física y/o sicológica de parte de sus padres o parientes.

Es excepcional que un niño sea atendido por Ml como motivo de consulta, por ello, el equipo de salud debe estar en condiciones de pesquisar los casos de maltrato y conocer las vías para proteger al niño en riesgo. Detectar o reconocer el $\mathrm{Ml}$ depende de los conocimientos y habilidades de una gran variedad de profesionales de la salud y su diagnóstico requiere de un acto médico de sospecha, el problema es que en pocas ocasiones las lesiones por maltrato son evidentes por lo tanto debe tenerse en cuenta los antecedentes personales y los signos clínicos que con mayor frecuencia hacen sospechar un caso de MI, es por esto que el odontólogo está en una posición clave para detectarlo (Lazenbatt \& Freeman, 2006) ya que más del $50 \%$ de los signos clínicos de Ml se presentan en boca, cara y cuello (American Academy of Pediatrics. Committee on Child Abuse and Neglect. American Academy of Pediatric Dentistry, 1999), más aún se considera a la cavidad oral como un foco central de MI debido a la importancia en la comunicación y nutrición (Ermertcan \& Ertan, 2010).

La negligencia es la forma más común de maltrato, corresponde al incumplimiento de los padres en buscar y seguir adelante con el tratamiento necesario para asegurar la salud oral de sus hijos. Una madre o padre negligente es aquel que después de haber sido advertido por el profesional de la importancia de la salud oral no lleva a su hijo a los controles periódicos con el odontólogo. Signos clínicos de negligencia son: caries irrestricta e infecciones recurrentes que pueden llevar a la pérdida de dientes (Kellogg \& Committee on Child Abuse and Neglect, 2005a).

Algunos signos clínicos de Maltrato Físico son contusiones, hemorragias, laceraciones, abrasiones y quemaduras en lengua, labios, mucosa bucal, paladar y encías (siendo la laceración de labio la más frecuente), además se describen los dientes decolorados que indican necrosis producto de traumatismos anteriores (Ermertcan \& Ertan).

Los signos clínicos de Maltrato sexual pueden ser: Petequias en la unión del paladar duro con el blando, herpes bucal, eritema inexplicable y mordidas humanas (Kellogg \& American Academy of Pediatrics Committee on Child Abuse and Neglect, 2005b).

Con respecto a las responsabilidades legales, el artículo 200 del Código Procesal Penal de Chile, indica: Lesiones corporales. Toda persona a cuyo cargo se encontrare un hospital u otroestablecimiento de salud semejante, fuere público o privado, dará en el acto cuenta al fiscal de la entradade cualquier individuo que tuviere lesiones corporales de significación, indicando brevemente el estadodel paciente y la exposición que hicieren la o las personas que lo hubieren conducido acerca del origende dichas lesiones y del lugar y estado en que se le hubiere encontrado. La denuncia deberá consignar elestado del paciente, describir los signos externos de las lesiones e incluir las exposiciones que hicieren elafectado o las personas que lo hubieren conducido.En ausencia del jefe del establecimiento, dará cuenta el que lo subrogare en el momento del ingresodel lesionado. El incumplimiento de la obligación prevista en este artículo se castigará con la pena que prevé elartículo 494 del Código Penal. Esto es complementado y clarificadocon el artículo 175: Denuncia Obligatoria: Estarán obligados a denunciar: Los jefes de establecimientos hospitalarios o de clínicas particulares y, en general, los profesionales en medicina, odontología, química, farmacia y de otras ramas relacionadas con la conservación o el restablecimiento de la salud, y los que ejercieren prestaciones auxiliares de ellas, que notaren en una persona o en un cadáver señales de envenenamiento o de otro delito

Teniendo el odontólogo conciencia y conocimiento de esto, podrá cumplir con el rol protector y defensor de los derechos del niño, ya que el MI compromete aquella calidad de vida digna de ser llamada humana y demanda del quehacer médico una obligación de asistencia y un actuar moral cuyo fundamento es la persona humana con su totalidad compleja y pluridimensional (Comité de Maltrato Infantil Sociedad Chilena de Pediatría, 2007).

La mayoría de las disciplinas de salud no incluye en sus planes de estudio y programas de educación contínua los aspectos de educación y formación relacionados con el maltrato, por lo tanto los profesionales no están preparados para ofrecer solución real a la salud de sus víctimas (Saliba et al., 2007).

\section{MATERIAL Y MÉTODO}

Se realizó una investigación de enfoque cuantitativo, de alcance exploratorio, descriptivo y relacional, con un diseño no experimental, transversal. Se confeccionó una encuesta que recogió: datos demográficos, 
estudios de postgrado, número de años de trabajo, número de niños atendidos a la semana y origen de la información recibida sobre MI.

Se evaluó la actitud según la sospecha y la denuncia de $\mathrm{Ml}$ y el conocimiento mediante 10 aseveraciones las cuales se respondían con verdadero o falso. La encuesta fue piloteada en un grupo pequeño de odontólogos y fue ajustada de acuerdo a la retroalimentación con el fin de mejorar el entendimiento.

Se incluyeron en el estudio los 9 centros asistenciales de salud de Concepción, Chile. Estos fueron: Hospital Clínico Regional Dr. Guillermo Grant Benavente, CESFAM (Centro de Salud Familiar) Pedro de Valdivia, CESFAM O'Higgins, CESFAM Víctor Manuel Fernández, CESFAM Tucapel, CESFAM Lorenzo Arenas, CESFAM Juan Soto Fernández, CESFAM Santa Sabina, CESFAM Villa Nonguén.

Se les pidió a cada odontólogo perteneciente a los centros asistenciales antes mencionados que respondiera la encuesta luego de haber sido informado sobre el objetivo y el protocolo de estudio, 53 (78\%) de los 68 odontólogos aceptaron.

Los datos fueron analizados utilizando estadística descriptiva y mediante las pruebas Chi cuadrado y Wilcoxon (Mann-Whitney $U$ ) con un 0,05 de significación. Para el análisis estadístico se usó el programa Infostat.

\section{RESULTADOS}

La población de estudio estuvo compuesta por 35 Mujeres (66\%) y 18 Hombres (34\%). $23(43,39 \%)$ Odontólogos con especialidad y $30(56,61 \%)$ generales, con un promedio de 19 años de trabajo ejerciendo la profesión, y un promedio de 26 niños atendidos a la semana.

Con respecto a la información sobre $\mathrm{MI}$, el 50,9\% declara haber recibido información sobre MI. El origen de esta información se representa en la Figura 1 donde se consideró como fuente formal a la recibida en la asistencia a congresos y cursos de capacitación y fuente informal a: lecturas de Internet, televisión y noticias de otros medios de comunicación.

Con respecto al conocimiento sobre $\mathrm{Ml}$, el promedio de respuestas correctas fue de 6,79 de un máximo de 10. La media de las respuestas correctas en los 35 odontólogos hombres fue de 7,09 y en las 18 mujeres odontólogas de 6,29.

Las aseveraciones realizadas tenían relación con: Aseveración Número 1: Definición de MI de la OMS. Aseveración Número 2: Signos Clínicos de Mordida Humana. Aseveración Número 3: Lugares principalmente afectados por el MI. Aseveración Número 4: Síntomas clínicos del MI.Aseveraciones Números 5 y 8: Datos de Sename 2010, sobre que género era el más abusador y abusado respectivamente. Asevera-

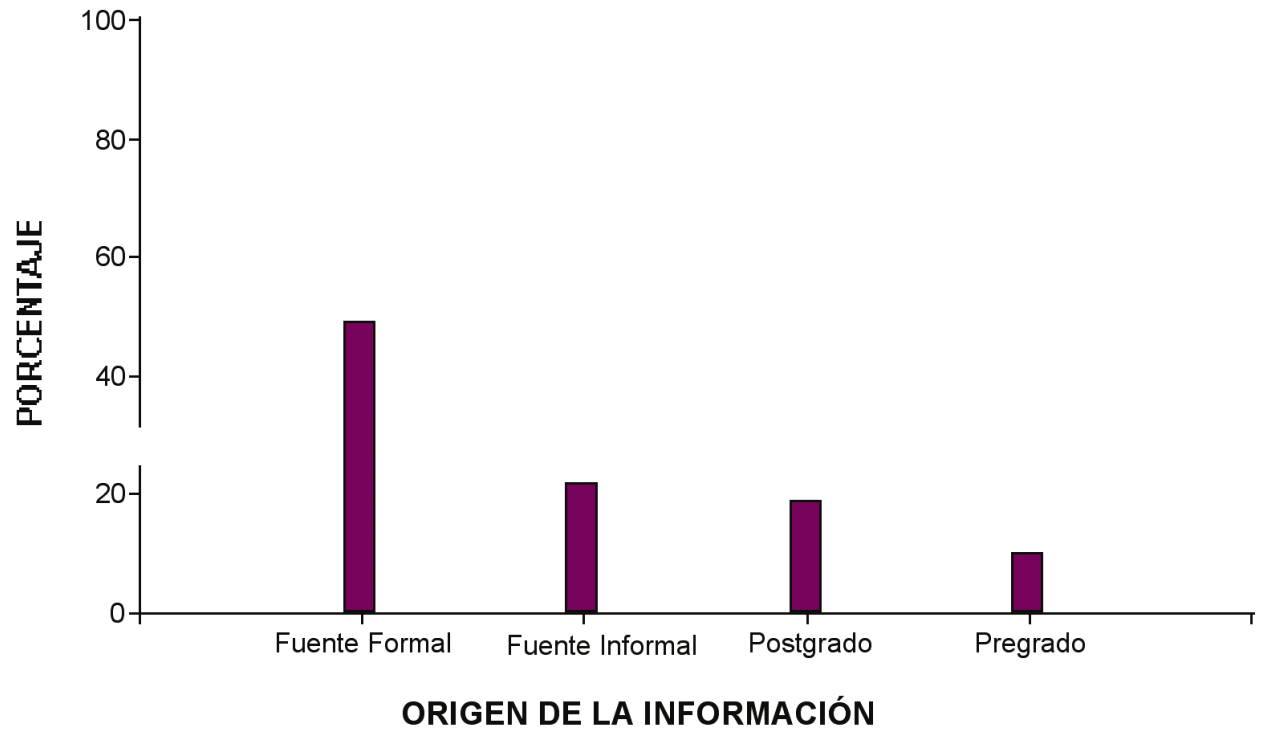

Fig. 1. Origen de la información recibida sobre MI y declarada por los odontólogos pertenecientes a los centros familiaresde salud de Concepción, Chile. 


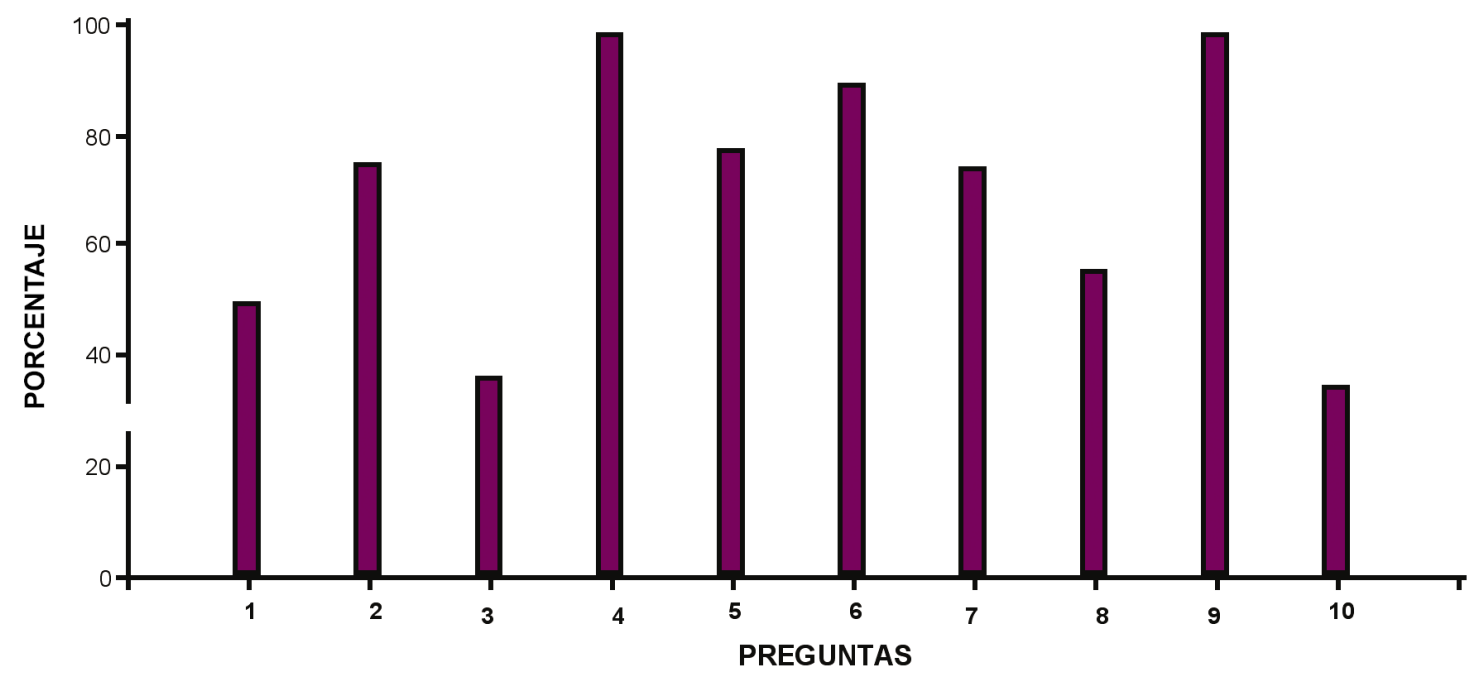

Fig. 2. Porcentaje de respuestas correctas por cada aseveración.

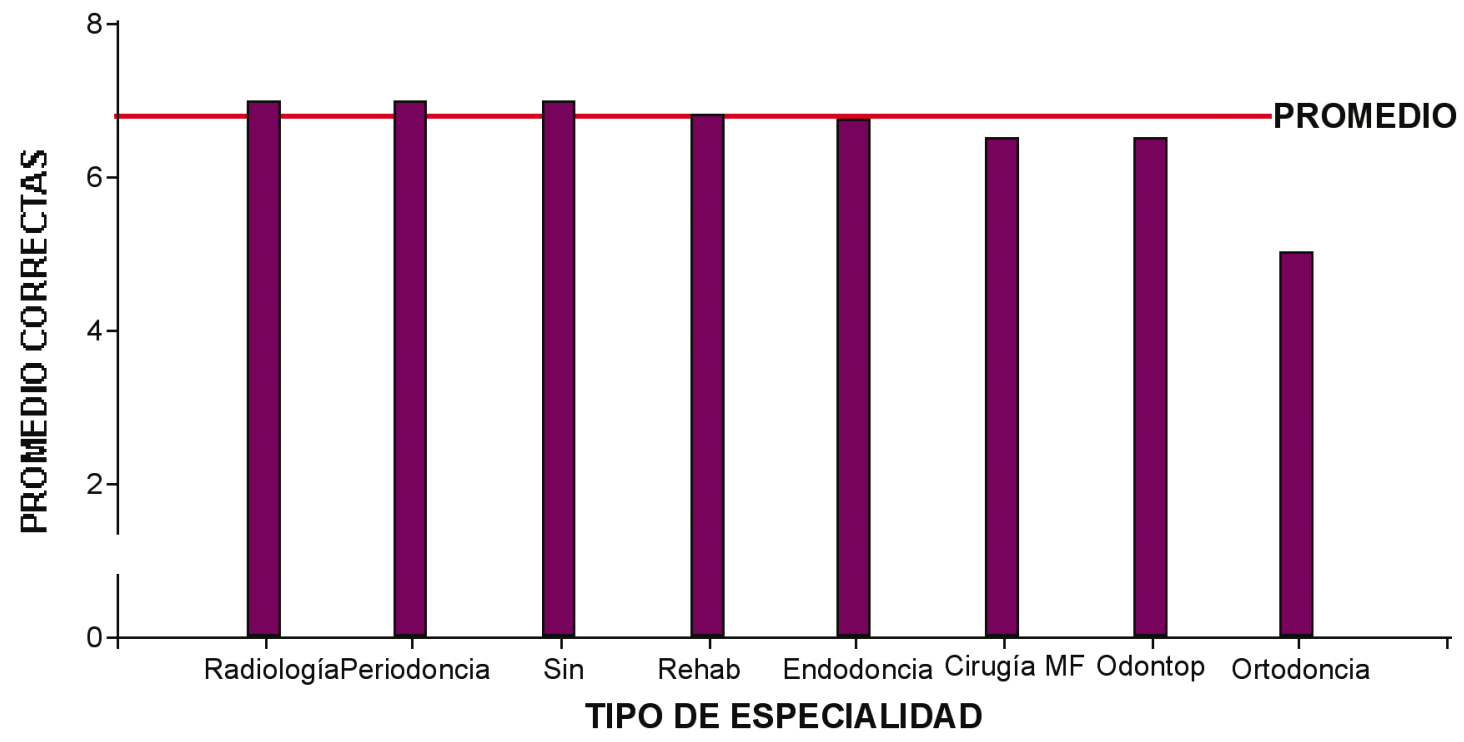

Fig. 3. Promedio de respuestas correctas según las especialidades.

ción Número 6: Signos Clínicos de Negligencia. Aseveraciones Números 7 y 9: Responsabilidades de la pesquisa y la denunciar sobre MI respectivamente.Aseveración Número 10: Signos clínicos de sexo oral forzado. Cada aseveración se respondía marcando una cruz en el recuadro $\mathrm{V}$ si era considerada verdadera y en el recuadro $\mathrm{F}$ si era considerada falsa.

El porcentaje de respuestas correctas por aseveración está descrito en la Figura 2. Con respecto a las aseveraciones más erradas, el $64 \%$ de los odontólogos no sabe que "más de la mitad de signos clínicos de MI están en boca, cara y cuello”, y el $66 \%$ no reconoce que "las petequias en el paladar podrían ser un signo clínico de sexo oral forzado".

El promedio de respuestas correctas por especialidad está detallado en la Figura 3. Los profesionales especialistas en radiología y periodoncia superaron el promedio general de respuestas correctas, al igual que los dentistas generales, por el contrario los especialistas en cirugía maxilofacial, odontopediatría y especialmente ortodoncia obtuvieron respuestas correctas inferiores a este.

Se encontraron diferencias significativas $(p=$ 
0,0326 ) en las variables de género con relación a conocimiento siendo mayor en las mujeres. Las variables,origen de la información recibida, número de años de trabajo, número de niños atendidos a la semana y especialidad resultaron independientes $(p>0,05)$ con respecto al número de respuestas correctas de conocimiento.

Con respecto a la actitud, 23 (43\%) odontólogos reconocen haber sospechado alguna vez de $\mathrm{MI}$, de ellos $16(70 \%)$ fueron mujeres y $7(30 \%)$ fueron hombres. De esos 23 odontólogos sólo $13(57 \%)$ han denunciado sus sospechas, de ellos $10(77 \%)$ fueron mujeres y $3(23 \%)$ fueron hombres. Las denuncias más frecuentes se dirigieron a: el/la asistente social o jefe del área del centro de salud y a carabineros. No hubo diferencia significativa al asociar actitud con información recibida, número de años de trabajo, número de niños atendidos a la semana, especialidad ni número de respuestas correctas.

\section{DISCUSIÓN}

Dentro del marco teórico de esta investigación se encontraron 5 trabajos de investigación relacionados con el tema, todos se realizaron mediante encuestas y se aplicaron a diferentes profesionales del área de la salud. En Turquía, se encuestó a médicos de atención primaria sobre el conocimiento y actitud del Ml y se concluyó que era inadecuado para el diagnóstico y la notificación del MI (Açik et al., 2004). Sin embargo en Porto Alegre, Brasil se evaluó a pediatras sobre el grado de conocimientos de $\mathrm{Ml}$ y se concluyó que sin tener estudios específicos sobre el tema, el grado de conocimiento fue suficiente, más de la mitad dijo que no confiaba en las agencias para proteger a los niños y el $94,6 \%$ informó temor a sanciones legales al denunciar a un caso de maltrato infantil (Pires et al., 2005).

Al evaluar y comparar a médicos, odontólogos y enfermeras en Irlanda del Norte se concluyó que los odontólogos presentaron las puntuaciones más bajas para identificar y reconocer el $\mathrm{Ml}$ y además se destacaron por su falta de voluntad en denunciarlos (Jouriles et al.). Así también tenemos que los encuestas hechas a odontólogos y estudiantes de odontología en Italia concluyen que el Ml es subestimado por los odontólogos, quienes tiene una mala actitud en la percepción del MI como patología. La mayoría son incapaces de hacer frente a los casos sospechosos, a pesar de que de ser conscientes de las responsabilidades médico legales de su trabajo. La negligencia y el descuido dental son los tipos menos conocidos y detectados de maltrato, a pesar de los los más frecuentes y la educación es clave para incrementar la capacidad de los dentistas para reconocer y denunciar el MI (Manea et al., 2007). Menos alentadora es la conclusión de un estudio realizado en DInamarca con dentistas e higienistas dentales, donde ambos grupos no cumplen suficientemente su rol protector infantil, ya que sólo el 33,9\% denunció sus sospecha de $\mathrm{Ml}$ a los servicios sociales. Sin embargo estos valores son mayores en los dentistas comparados con los higienistas y para los empleados del servicio de salud pública versus los privados, debido probablemente a la diferencia de educación familiar y al bajo número de niños que se atiende en consultas privadas (Uldum et al., 2011). Si bien ninguna de estas investigaciones son comparables se puede observar una similitud en diversos países en relación con la actitud hacia el tema en el área odontológica.

En nuestro estudio se estableció que en general el conocimiento es satisfactorio. Sin embargo los dentistas generales no demostraron estar preparados para identificar el MI, ya que la información con la que cuentan no refuerza su detección clínica. De 58 odontólogos, 23 reconocen haber sospechado alguna vez de Ml, y de éstos, sólo 13 han denunciado sus sospechas.

Los especialistas (ortodoncistas y odontopediatras) que tienen atención exclusiva de niños revelaron conocimientos inferiores al promedio. $Y$ si bien los dentistas generales se encontraron sobre el promedio de respuestas correctas, el origen de la fuente de información sobre Ml no se encontró en el pregrado de la carrera de Odontología, teniendo en cuenta que la mayor cantidad de odontólogos que trabajan en los servicios de salud públicos de Concepción no poseen estudios de posgrado, por ello se plantea entonces, la necesidad de incorporar o reforzar el tema del MI en las asignaturas de pregrado en las facultades de odontología de la zona.

Se encontró diferencia significativa en las variables de género con relación a conocimiento siendo mayor en las mujeres. Las variables de información recibida, número de años de trabajo, número de niños atendidos a la semana y especialidad resultaron independientes con respecto al número de respuestas correctas de conocimiento.

No se encontró un instrumento universal y reconocido de medición de conocimiento de MI, por lo que se propone a futuro generarlo. 
La validación de instrumentos de evaluación de conocimiento y actitudes sobre maltrato infantil, así como, ampliar y profundizar estudios del tema contri- buirá a mejorar la actitud del odontólogo en su responsabilidad como profesional del área de la salud frente a la protección infantil.

FIERRO, M. C.; SALAZAR, S. E.; RUIZ, S. L.; LUENGO, M. L. \& PÉREZ, F. A. Attitude towards child abuse according to the knowledge of dentists in Concepción, Chile. Int. J. Odontostomat., 6(1):105-110, 2012.

ABSTRACT: Child abuse (CA) is considered to be a global public health problem, it manifests in all countries and in all social and religious groups. The role of dentists specialized in children involves a close and frequent relationship providing privileged circumstances for the detection of CA. The purpose of our study is to describe dentist attitudes and awareness concerning CA at nine health care centers in Concepción, Chile. A quantitative, descriptive and transversal exploratory analysis was performed by implementing a survey that collected demographic data, awareness, attitudes, suspicions and accusations of CA. The population size of the study consisted of 53 dentists. Chi square and Wilcoxon (Mann-Whitney U) were used as statistical tests. Largely, awareness was satisfactory. The variables of sex and awareness were significantly higher in women ( $p$ $=0.0326$ ). However, no differences were found among sex and attitudes. The information received by the dentists during their training, and the number of years of work experience did not correlate with awareness. The undergraduate training of the dentists did not provide any information on CA. This emphasizes the need to incorporate or reinforce the aspect of CA in undergraduate courses taught at the Faculties of Dentistry in Concepción, Chile.

KEY WORDS: child abuse, knowledge, attitude, dentists.

\section{REFERENCIAS BIBLIOGRÁFICAS}

Açik, Y.; Deveci, S. E. \& Oral, R. Level of knowledge and attitude of primary care physicians in Eastern Anatolian cities in relation to child abuse and neglect. Prev. Med., 39(4):791-7, 2004.

American Academy of Pediatrics. Committee on Child Abuse and Neglect. American Academy of Pediatric Dentistry. Oral and dental aspects of child abuse and neglect. Ad Hoc Work Group on Child Abuse and Neglect. Pediatrics, 104(2 Pt.1):348-50, 1999.

Comité de Maltrato Infantil Sociedad Chilena de Pediatría. El Maltrato Infantil desde la bioética: El sistema de salud y su labor asistencial ante el maltrato infantil, ¿qué hacer?. Rev. Chil. Pediatr., 78(Supl. 1):85-95, 2007.

Ermertcan, A. T. \& Ertan, P. Skin manifestations of child abuse. Indian J. Dermatol. Venereol. Leprol., 76(4):317-26, 2010.

Jouriles, E. N.; McDonald, R.; Slep, A. M.; Heyman, R. E. \& Garrido, E. Child abuse in the context of domestic violence: prevalence, explanations, and practice implications. Violence Vict., 23(2):221-35, 2008.

Kellogg, N. D. \& Committee on Child Abuse and Neglect. The Evaluation of Sexual Abuse in Children. Pediatrics, 116(2):506-12, 2005a.

Kellogg, N, \& American Academy of Pediatrics Committee on Child Abuse and Neglect. Oral and dental aspects of child abuse and neglect. Pediatrics, 116(6):1565-8, 2005b.
Lazenbatt, A. \& Freeman, R. Recognizing and reporting child physical abuse: a survey of primary healthcare professionals. J. Adv. Nurs., 56(3):227-36, 2006.

Manea, S.; Favero, G. A.; Stellini, E.; Romoli, L.; Mazzucato, M. \& Facchin, P. Dentists' perceptions, attitudes, knowledge, and experience about child abuse and neglect in northeast Italy. J. Clin. Pediatr. Dent., 32(1):1925, 2007.

Pires, J.; Zubaran, M.; Vieira, E.; Nava, T.; Feldens, L.; Castilhos, K.; Simas, V. \& Franzon, N. Barriers for reporter of child abuse by pediatricians. Rev. Bras. Saude Mater. Infant., 5(1):103-8, 2005.

Saliba, O.; Garbin, C. A.; Garbin, A. J. \& Dossi, A. P. Responsibility of health providers in domestic violence reporting. Rev. Saude Publica, 41(3):472-7, 2007.

Uldum, B.; Christensen, H. N.; Welbury, R. \& Poulsen, S. Danish dentists' and dental hygienists' knowledge of and experience with suspicion of child abuse or neglect. Int. J. Paediatr. Dent., 20(5):361-5, 2011.

UNICEF. Maltrato infantil y relaciones familiares en Chile. Análisis comparativo 1994 - 2000 - 2006. Santiago, Chile, Andros Impresores, 2008.

Dirección para correspondencia:

Prof. Dra. Claudia Fierro Monti

Depto. de Pediatría Bucal

Facultad de Odontología

Universidad de Concepción

Concepción

CHILE

Email: posgradofao@udec.cl
Recibido : 02-01-2011

Aceptado: 11-02-2012 